\title{
Topical ofloxacin compared with gentamicin in the treatment of external ocular infection
}

\author{
Arlene Gwon for the Ofloxacin Study Group ${ }^{\star}$
}

\begin{abstract}
In a double-masked, randomised, controlled study the effectiveness and safety of $0.3 \%$ ofloxacin solution were compared with those of $0.3 \%$ gentamicin ophthalmic solution in treating external bacterial ocular infections. The clinical improvement rate for patients treated with ofloxacin was $98 \%(51 / 52)$ and $92 \%(48 / 52)$ for those treated with gentamicin. Microbiological improvement was achieved in $78 \%(40 / 51)$ of the ofloxacin patients, compared with $67 \%(35 / 52)$ of the gentamicin group. Ofloxacin treatment eradicated or controlled $85 \%(86 / 101)$ of the Gram positive and $89 \%(17 / 19)$ of the Gram negative organisms cultured, compared with $83 \%(103 / 124)$ and $78 \%$ (29/37), respectively, after gentamicin treatment. None of these differences were statistically significant. The incidence of adverse effects attributable to ofloxacin treatment $(3 \cdot 2 \%)$ was less than that reported for gentamicin $(7 \cdot 1 \%)$. Ofloxacin proved to be an effective, safe, and comfortable therapy for external bacterial ocular infection.

(Brf Ophthalmol 1992; 76: 714-718)
\end{abstract}

The aminoglycosides gentamicin and tobramycin are well established as first-line therapy for external ocular infections, and possess a broad spectrum of activity against Gram positive and Gram negative organisms. ${ }^{1-3}$ However, resistance to these antibiotics is increasing. For example, resistance to topical aminoglycoside therapy may be encountered in as many as $8 \%$ to $10 \%$ of ulcerative keratitis cases caused by Pseudomonas aeruginosa. ${ }^{4}$ Resistance appears to be even greater in ocular infections caused by Gram positive organisms. ${ }^{5}$ Adverse drug effects such as punctate epithelial keratitis are encountered in some patients. ${ }^{3}$ The problems of resistance to the aminoglycosides and the possibility of adverse responses to these agents in some patients have given impetus to the search for new ocular anti-infective agents.

Ofloxacin is among the more promising agents under investigation as a treatment for ocular infections. ${ }^{6}$ An in vitro study demonstrated that ofloxacin is more potent than non-quinolone antibiotics (gentamicin, tobramycin, chloram-

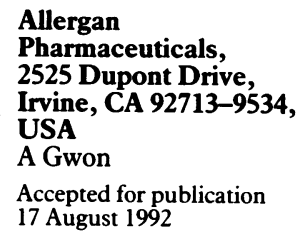

^Ofloxacin Study Group I Investigators: Sanford Rakofsky, MD, Coral Gables, FL; Theodore Buka, MD, Cincinnati, OH; Valerie Moore, MD, Fort Myers, FL; Penny Asbell, MD, New York, NY; Beth Friedland, MD, Research Triangle Park, NC; Lawrence Lohman, MD, Kent, OH; William J Faulkner, MD, Reading, $\mathrm{OH}$; J Elliott Blaydes, MD, Bluefield, West Virginia; Bruce Koffer MD , Lexington KY; James V Aquavella, MD, Bruce Koffler, MD, Lexington, KY; James $\mathrm{V}$ Aquavella, MD Rochester, NY; Leonard R Borrmann, Pharm D, Arlen Gwon, MD, and Jess Frank, PhD (biostatistician): Allergan
Pharmaceuticals, Irvine, CA, USA. phenicol, polymyxin B) and more potent than the quinolone, norfloxacin, against various ocular species, including Staphylococcus aureus, Staphylococcus epidermidis, and Streptococcus pneumoniae. ${ }^{7}$ In another recent in vitro study comprising five fluoroquinolones (ciprofloxacin, norfloxacin, ofloxacin, pefloxacin, and temafloxacin) with gentamicin, tobramycin, and cefazolin, the fluoroquinolones had excellent in vitro activity against isolates of common bacterial pathogens, and were less toxic to the corneal epithelium than the aminoglycosides. ${ }^{8}$ Ofloxacin also shows potent activity in vitro against chlamydial species, various anaerobes, and other species..$^{9-24}$

Fluoroquinolones, such as ofloxacin, derive their antibacterial activity from their ability to inhibit bacterial DNA gyrase, an enzyme that catalyses the conversion of relaxed covalently closed circular DNA to a supercoiled form. ${ }^{25}$ These compounds alter the structure and disrupt the function of bacterial DNA and interfere with fundamental processes, including DNA replication, recombination, repair, and transcription. Ofloxacin-resistant bacterial strains do not form readily in vitro, and when such strains do appear they generally are not as viable as ofloxacinsensitive strains. ${ }^{26} 27$ Cross resistance with ofloxacin and other classes of antibiotics has not been reported.

In clinical studies conducted to date, topical ofloxacin has significantly reduced the clinical signs and symptoms of ocular infection and eradicated the causative organism in a high proportion of patients. ${ }^{28}$ The present study was designed to assess the clinical value of topical ofloxacin treatment by comparing its efficacy, safety, and comfort of application with those of standard topical gentamicin therapy.

\section{Subjects and methods}

\section{PATIENTS}

A total of 194 patients with suspected external ocular bacterial infection, including conjunctivitis, blepharitis, and blepharoconjunctivitis,

\section{Table 1 Exclusion criteria}

Patients were excluded from the study if any of the following were present:

(1) Active ophthalmic disease (except external bacterial infection)

(2) Uncontrolled systemic disease, such as hypertension or diabetes

(3) Use of other topical ophthalmic medications

(4) Use of concomitant systemic antibiotics

(5) Sensitivity to test medications or solution components

(5) Pregnancy or nursing, or planned pregnancy during the study 
were enrolled at 18 study sites. All patients signed informed consent forms describing the design and purpose of the study as well as the potential risks of participation. None of the patients were known to be sensitive to the test medications or solution constituents, such as benzalkonium chloride, or to nalidixic acid, gentamicin, or any other aminoglycoside. These and other exclusion criteria are shown in Table 1.

\section{DRUG TREATMENT}

Patients were randomly assigned to receive either $0.3 \%$ ofloxacin $(n=93)$ or $0.3 \%$ gentamicin $(\mathrm{n}=98)$, administered in identical masked bottles. One drop of either ofloxacin or gentamicin solution was applied to the affected eye(s) six times daily (every 2 to 4 hours) for 2 days (day 1 and day 2) and then four times daily for the next 8 days (day 3 to day 10). The first dose was administered by the investigator, and all subsequent doses were self-administered by the patient. Patients were instructed to discontinue use of the study medication at least 12 hours prior to the second follow-up examination on day 11 .

PATIENT EXAMINATION, CULTURE EVALUATION During a baseline examination, medical and ophthalmic histories were taken, visual acuity tested, biomicroscopy and ophthalmoscopy performed, symptoms of ocular infection assessed, and conjunctival and eyelid specimens obtained. Sensitivity testing of the clinical isolates for ofloxacin, gentamicin, and tobramycin was performed with the Kirby Bauer disc diffusion method. The patients were re-examined on days 3 to 5 and again on day 11. The follow-up examinations on day 11 consisted of the same clinical observations as the baseline examinations and also included the investigator's evaluation of the effectiveness of the treatment and an assessment of the comfort of the drug treatment. In patients with positive baseline culture findings, follow-up culture tests were performed at the day 11 examination.

At all follow-up examinations, the safety of the drug treatments was evaluated by noting adverse reactions and changes from baseline in visual acuity, ophthalmoscopic findings, and lens pathology. Comfort of the test medications was evaluated based on the severity of burning, stinging, tearing, itching, foreign body sensation, photophobia, blurring of vision, dryness,

Table 2 Drug efficacy assessment ${ }^{2}$

\begin{tabular}{|c|c|}
\hline $\begin{array}{l}\text { Colony-forming units } \\
\text { per swab }\end{array}$ & Bacterial species \\
\hline $\begin{array}{l}\text { More than } 0 \\
\text { (Class A) }\end{array}$ & $\begin{array}{l}\text { Group A streptococci } \\
\text { Streptococcus pneumoniae } \\
\text { All Gram negative rods } \\
\text { Neisseria sD. }\end{array}$ \\
\hline $\begin{array}{l}\text { More than } 10 \\
\text { (Class B) }\end{array}$ & $\begin{array}{l}\text { Alpha-haemolytic streptococci } \\
\text { Staphylococcus aureus } \\
\text { Other micrococcaceae } \\
\text { Branhamella catarrhalis }\end{array}$ \\
\hline $\begin{array}{l}\text { More than } 100 \\
\text { (Class C) } \\
\text { More than } 10000 \\
\text { (Class D) }\end{array}$ & $\begin{array}{l}\text { Staphylococcus epidermidis } \\
\text { Bacillus sp. } \\
\text { Corynebacterium sp. and diphtheroids }\end{array}$ \\
\hline
\end{tabular}

and pain experienced after applying the study medication.

To obtain ocular cultures, standard calcium alginate swabs were moistened with sterile unpreserved saline and rolled along the lid margins and conjunctival cul-de-sacs of each affected eye. Separate lid and conjunctival cultures were taken for each eye. Each culture swab was placed in a sterile tube with $1.0 \mathrm{ml}$ of transport-dissolving buffer, and was delivered to the microbiological laboratory within 4 hours of collection.

For quantitative analysis in the laboratory, the tube was vortexed until the swab fibres were finely dispersed. A sample of the suspension was plated directly on to a $5 \%$ horse blood agar plate and a chocolate agar plate. A dilution (1:10) of the sample was prepared in sterile trypticase soy broth and was also plated onto both blood and chocolate agar plates. Colony-forming units were counted after all plates were incubated for 48 hours at $37^{\circ} \mathrm{C}$ in $4 \% \mathrm{CO}_{2}$. Bacteria were identified and sensitivity tests (Kirby Bauer disc diffusion method) to ofloxacin, tobramycin, and gentamicin were performed.

\section{DATA ANALYSIS}

Drug efficacy assessment was restricted to patients with positive baseline culture results, based on the criteria of Cagle $e t a l^{3}$ (Table 2). Baseline culture findings were positive in 52 of 93 subjects $(56 \%)$ treated with ofloxacin and in 53 of $98(54 \%)$ subjects in the gentamicin group.

The microbiological improvement rate - that is, the proportion of patients evaluable for efficacy who had improved microbiologically, was determined by evaluating the change from baseline in bacterial colony counts. Microbiological outcomes, based on the worst outcome of all species at all culture sites, were classified as follows: proliferated (bacterial colony count positive, based on species-specific criteria listed above and greater than baseline); no change (bacterial colony count equal to baseline); reduced (bacterial colony count less than baseline but still greater than the species-specific threshold listed above); controlled (bacterial colony count less than the species specific threshold listed above); or eradicated (bacterial colony count equals zero). Patients were considered to have improved microbiologically if

Table 3 Clinical improvement rate: 10 key variables and calculation of clinical improvement

Calculating the clinical improvement rate:

The clinical improvement rate was derived from clinical evaluation scores of the $10 \mathrm{key}$ variables listed below, using a 4-point scale: $0=$ none; $1=$ mild; $2=$ moderate; and $3=$ severe 4-point scale: $0=$ none; $1=$ mild; $2=$ moderate; and $3=$ severe.
The cumulative summary score, used to gauge clinical outcome, was calculated as the sum of the scores for the 10 variables.

Key variables

Lid erythema Conjunctival oedema

Lid oedema

Lid crusting

Palpebral conjunctival hyperaemia Conjunctival discharge

Bulbar conjunctival hyperaemia

Corneal oedema

Foreign body sensation

Clinical outcome

Definition

Resolved

Diminished

No change

Worsened

Cumulative summary score value
0
< baseline and $>0$
= baseline and $>0$
$>$ baseline or patient discontinued due to lack
of drug efficacy


the bacterial infection was either eradicated or controlled.

The clinical improvement rate was defined as the proportion of patients evaluable for efficacy who showed improvement in a cumulative summary score involving 10 key biomicroscopy and symptom variables (Table 3 ).

Patients were considered to have improved overall if they had improved both clinically and microbiologically. The statistical significance of differences in the clinical, microbiological, and overall improvement rates between the ofloxacin and gentamicin groups was determined by the Cochran-Mantel-Haenszel test, with stratification by investigator. ${ }^{29}$ This stratified analysis was completed in an attempt to adjust for differences between investigators at the 18 sites, particularly regarding clinical scoring.

\section{Results}

Three of the 194 patients enrolled in the study were disqualified prior to receiving medication. Treatment safety was evaluated in the remaining

Table 4 Demographic profile

\begin{tabular}{lll}
\hline & Ofloxacin group & Gentamicin group \\
\hline $\begin{array}{l}\text { Age (mean, years) } \\
\text { Sex: }\end{array}$ & $59 \cdot 4$ & $54 \cdot 8$ \\
$\quad$ Male & $25(48 \cdot 1 \%)$ & $15(28 \cdot 3 \%)$ \\
Female & $27(51 \cdot 9 \%)$ & $38(71 \cdot 7 \%)$ \\
Race: & & \\
White & $44(84 \cdot 6 \%)$ & $38(71 \cdot 7 \%)$ \\
Black & $8(15 \cdot 4 \%)$ & $15(28 \cdot 3 \%)$ \\
Iris colour: & & \\
Blue & $13(25 \cdot 5 \%)$ & $19(34 \cdot 8 \%)$ \\
Green & $5(9 \cdot 8 \%)$ & $2(3 \cdot 8 \%)$ \\
Brown & $27(52 \cdot 9 \%)$ & $29(54 \cdot 7 \%)$ \\
Hazel & $6(11 \cdot 8 \%)$ & $3(5 \cdot 7 \%)$ \\
Data missing & $1(-)$ & $0(-)$ \\
& & \\
& &
\end{tabular}

Table 5 Clinical diagnoses

\begin{tabular}{lcc}
\hline & Ofloxacin & Gentamicin \\
\hline Conjunctivitis & $47(90 \cdot 4 \%)$ & $48(90 \cdot 6 \%)$ \\
Blepharitis & $0(0 \%)$ & $2(3 \cdot 8 \%)$ \\
Blepharoconjunctivitis & $2(3 \cdot 8 \%)$ & $1(1.9 \%)$ \\
Keratitis & $1(1.9 \%)$ & $0(0 \%)$ \\
Keratoconjunctivitis & 0 & $1(1.9 \%)$ \\
Corneal ulcer & $2(3 \cdot 84 \%)$ & $1(1.9 \%)$ \\
\hline
\end{tabular}

Table 6 Clinical, microbiological, and overall improvement rates in patients treated with ofloxacin or gentamicin

\begin{tabular}{llll}
\hline & \multicolumn{4}{l}{ Number (\%) improved } \\
\cline { 2 - 4 } Drug treatment & Clinical & Microbiological & Overall \\
\hline Ofloxacin & $51 / 52(98)$ & $40 / 51(78)$ & $40 / 51(78)$ \\
Gentamicin & $48 / 52(92)$ & $35 / 52(67)$ & $32 / 51(63)$
\end{tabular}

There were no statistical differences between the groups in any of the improvement rates, though there was a trend $(p=0.089)$ in the overall improvement results favouring ofloxacin.

Table 7 Microbiological improvement rates for infections involving selected microbial species

\begin{tabular}{llllll}
\hline & \multicolumn{2}{l}{ Number of patients } & & \multicolumn{2}{c}{ Percent improved } \\
\cline { 2 - 3 } Organism & Ofloxacin & Gentamicin & & Ofloxacin & Gentamicin \\
\hline Acinetobacter anitratum & 4 & 4 & 100 & 50 \\
Acinetobacter calcoaceticus lwoff & 2 & 2 & & 100 & 100 \\
Proteus mirabilis & 4 & 7 & & 100 & 100 \\
Micrococcus species & 6 & 11 & & 33 & 54 \\
Pseudomonas aeruginosa & 1 & 2 & & 100 & 0 \\
Staphylococcus aureus & 24 & 15 & & 88 & 87 \\
Staphylococcus epidermidis & 55 & 66 & & 87 & 85 \\
Staphylococcus species & 8 & 11 & & 88 & 82 \\
Streptococcus viridans & 4 & 3 & & 100 & 100 \\
\hline
\end{tabular}

191 patients: 93 were treated with ofloxacin and 98 with gentamicin.

A total of 105 subjects ranging in age from 19 to 88 years were considered evaluable for efficacy because of positive baseline cultures. Of these, 25 men and 27 women were assigned by random selection to receive ofloxacin, while 15 men and 38 women were designated for treatment with gentamicin. The two treatment groups were not significantly different with regard to age, race, iris colour, or medical and ophthalmic histories, though there were significantly more women than men in the gentamicin group (Table 4).

A significantly greater proportion of the gentamicin patients presented with a history of corneal disease compared with the ofloxacin group; otherwise, there were no significant differences in clinical diagnoses between the treatment groups (Table 5). Conjunctivitis was the most common diagnosis among the study subjects, accounting for $90 \%$ of culture-positive patients. Another $5 \%$ of patients were diagnosed with blepharoconjunctivitis and blepharitis. There were no significant differences between the ofloxacin and gentamicin groups with respect to the frequency of specific diagnoses.

Among patients treated with ofloxacin, 98\% (51/52) were either clinically cured or improved by day 11 , compared with $92 \%(48 / 52)$ of the gentamicin group (Table 6). The signs and symptoms of infection were judged to be completely resolved in $52 \%$ (27/52) of the ofloxacin group, compared with $44 \%(23 / 52)$ of the gentamicin group at day 11 . There were no notable differences in clinical improvement rates between patients with different baseline diagnoses. Ninety eight percent of ofloxacin-treated subjects with conjunctivitis were found to have improved by day 11 , compared with $100 \%$ of those with other diagnoses. Among the gentamicin group, $91 \%$ and $100 \%$ of the patients with conjunctivitis and other diagnoses, respectively, had improved by day 11 . None of the differences between the groups showed statistical significance.

\section{MICROBIOLOGICAL IMPROVEMENT}

Microbiological improvement was achieved in $78 \%(40 / 51)$ of the ofloxacin patients, compared with $67 \%(35 / 52)$ of the gentamicin group (Table 6 ), although the difference was not statistically significant. Ofloxacin treatment eradicated the infecting bacteria in $67 \%(34 / 51)$ of patients at day 11 , compared with $58 \%(30 / 52)$ after gentamicin treatment. Proliferation occurred in $16 \%(8 / 51)$ of the ofloxacin group vs $27 \%(14 / 52)$ of gentamicin-treated subjects. Nonetheless, all the ofloxacin-treated patients in whom proliferation had occurred were found to have improved clinically as of day 11 , compared with 12 of 14 gentamicin patients who had clinical improvement by this time.

Ofloxacin treatment eradicated or controlled $85 \%(86 / 101)$ of the Gram positive organisms and $89 \%(17 / 19)$ of the Gram negative organisms cultured, compared with $83 \%(103 / 124)$ and $78 \%$ (29/37), respectively, after gentamicin treatment. In cases involving the most virulent organisms (for example, those with threshold 
values of 0 as defined by Cagle $e t$ al $^{3}$ ), eradication or control was achieved in $87 \%(14 / 16)$ of the ofloxacin patients and in $82 \%(36 / 44)$ of the gentamicin group. Microbiological improvement rates for selected micro-organisms cultured during the study are listed in Table 7.

Disc diffusion sensitivity testing revealed that $2 \%(4 / 195)$ of the Gram positive organisms isolated were resistant to ofloxacin; $11 \%(22 / 197)$ were resistant to gentamicin; and 18\% (34/194) were resistant to tobramycin. None of the Gram negative isolates were found to be resistant to ofloxacin or gentamicin; however, $12 \%(5 / 41)$ of the Gram negative isolates were resistant to tobramycin.

Among the ofloxacin patients, $78 \%$ (40/51) improved overall - that is, both clinically and microbiologically, compared with $63 \%$ (32/51) of gentamicin patients (Table 6). The observed differences in clinical, microbiological, or overall improvement rates between the ofloxacin and gentamicin groups were not statistically significant.

Adverse reactions possibly caused by drug treatment were encountered in three of the 93 ofloxacin patients $(3 \cdot 2 \%)$ and seven of the 98 gentamicin patients $(7 \cdot 1 \%)$. These reactions, which included burning, stinging, and photophobia, necessitated discontinuation of the drug. No drug treatment related effects on visual acuity, ophthalmoscopic findings, or lens pathology were observed. There was no notable difference between treatment groups in comfort of drug application.

\section{Discussion}

The results of this study indicate that $0.3 \%$ ofloxacin is an effective, safe, and comfortable treatment for external ocular bacterial infection, primarily conjunctivitis and blepharoconjunctivitis. The clinical, microbiological, and overall improvement rates achieved with ofloxacin were $6 \%, 11 \%$, and $15 \%$ higher, respectively, than those with gentamicin, though statistical significance could not be established.

The clinical and microbiological improvement rates observed in the present study for ofloxacin (98\% and $78 \%$, respectively) and for gentamicin ( $92 \%$ and $67 \%$, respectively) are consistent with the results of earlier clinical studies. ${ }^{23}{ }^{28}{ }^{30}$ Mitsui et al found that topical ofloxacin produced a good or excellent response in $93 \%$ of patients. ${ }^{28}$ Cagle et al observed clinical cure or improvement in $92 \%$ of patients treated with topical gentamicin. ${ }^{3}$ Gentamicin yielded microbiological improvement rates of $84 \%$ and $74 \%$, respectively, for infections of the conjunctiva and skin-lash margin. ${ }^{3}$

Two percent of Gram positive isolates and no Gram negative isolates were resistant to ofloxacin, which is consistent with previous in vitro reports indicating that strains resistant to this fluoroquinolone do not readily form. ${ }^{26} 27$ Since the mechanism of action of ofloxacin involves interference with the supercoiling of chromosomal DNA, rather than a plasmidmediated process, resistance would not be expected to be easily transferred. In contrast, increasing resistance to the aminoglycosides has been reported. ${ }^{45}$ In the present study the proportion of Gram positive isolates resistant to gentamicin was approximately five times greater than with ofloxacin. The proportion of Gram positive isolates resistant to tobramycin was nine times greater. Further, a substantial proportion of Gram negative isolates (12\%) were resistant to tobramycin, whereas no Gram negative isolates resistant to ofloxacin were observed.

The incidence of side effects owing to ofloxacin was relatively low $(3 \cdot 2 \%)$ in our study. A low incidence of side effects $(1 \cdot 1 \%)$ was also observed in ofloxacin-treated patients by Mitsui and coworkers. ${ }^{28}$

The present demonstration of the effectiveness and safety of ofloxacin may be at least partially explained by recent animal and human studies showing that effective dosages of this drug are achieved and maintained after topical administration and that the risks of systemic or other side effects is low. In rabbits, tear ofloxacin levels exceeded the minimal inhibitory concentration against $90 \%$ of bacterial strains tested in vitro $\left(\mathrm{MIC}_{90}\right)$ for 4 hours after topical application, whereas gentamicin levels remained higher than the $\mathrm{MIC}_{90}$ against $\mathrm{Gram}$ positive organisms for only 20 minutes and the $\mathrm{MIC}_{90}$ against Gram negative organisms for only 120 minutes. ${ }^{31}$ In addition, 4 hours after topical application, mean ofloxacin levels in human tear film $(9 \cdot 16(\mathrm{SD} 8 \cdot 24) \mu \mathrm{g} / \mathrm{g})^{31}$ were higher than the $\mathrm{MIC}_{90}(2 \mu \mathrm{g} / \mathrm{ml})$ for ofloxacin reported in the literature. ${ }^{24}$

In another study, topical $0.3 \%$ ofloxacin ointment produced higher tear concentrations for a longer period than did an aqueous solution of the drug. ${ }^{33}$ The highest tear level obtained with the ointment was detected within 30 minutes, and effective concentrations were present 60 minutes after administration.

The fluoroquinolone, ofloxacin, is a promising new agent for treating external ocular infection and may prove especially valuable in cases that are difficult to manage because of resistance to standard agents now in use.

Results of this study were presented as a poster at the annual meeting of the American Academy of Ophthalmology, October

This study was sponsored by Allergan, Inc, Irvine, California. Allergan is investigating the clinical utility of topical ofloxacin in the treatment of ocular infections.

1 Jarudi N, Golden B, Hoyme J, Tyson MD, Harter JG Comparison of antibiotic therapy in presumptive bacterial conjunctivitis. Am $\mathcal{F}$ Ophthalmol 1975; 79: 790-4.

2 Cagle GD, Abshire RL. Quantitative ocular bacteriology: a method for the enumeration and identification of bacteria from the skin-lash margin and conjunctiva. Inves Ophthalmol Vis Sci 1981; 20: 751-7.

3 Cagle G, Davis S, Rosenthal A, Smith J. Topical tobramycin and gentamicin sulfate in the treatment of ocular infections: multicenter study. Curr Eye Res 1981/1982; 1: 523-34.

4 Gelender H, Rettich C. Gentamicin-resistant Pseudomonas aeruginosa corneal ulcers. Cornea 1984; 3: 21-6.

5 Mehta NJ, Webb RM, Krohel GB, Smith RS. Clinical inefficacy of tobramycin and gentamicin sulfate in the treatment of ocular infections. Cornea 1984; 3: 228.

6 Borrmann LR, Leopold IH. The potential use of quinolones in future ocular antimicrobial therapy. Am F Ophthalmol 1988; 104: $227-9$.

7 Osato MS, Jensen HG, Trousdale MD, Bosso JA, Borrmann LR, Frank J, et al. The comparative in vitro activity of ofloxacin and selected ophthalmic antimicrobial agents against ocular bacterial isolates. Am F Ophthalmol 1989; 108: against

8 Cutarelli PE, Lass JH, Lazarus HM, Putman SC, Jacobs MR. Topical fluoroquinolones: antimicrobial activity and in vitro corneal epithelial toxicity. Curr Eye Res 1991; 10: 557-63. 9 Sato K, Matsuura Y, Inoue M, Tsutomu U, Yasuaki O, 
Hidemasa $\mathrm{O}$, et al. In vitro and in vivo activity of DL-8280, a new oxazine derivative. Antimicrob Agents Chemother 1982 22: $548-53$.

10 Chantot JF, Bryskier A. Antibacterial activity of ofloxacin and other 4-quinolone derivatives: in-vitro and in-vivo comparison. F Antimicrob Chemother 1985; 16: 475-84.

11 King A, Shannon K, Phillips I. The in-vitro activities of enoxacin and ofloxacin compared with that of ciprofloxacin $\mathcal{F}$ Antimicrob Chemother 1985; 15: 551-8.

12 Kumada T, Neu HC. In-vitro activity of ofloxacin, a quinolone carboxylic acid compared to other quinolones and other antimicrobial agents. I Antimicrob Chemother 1985; 16: 563-74.

13 Ridgway GL, O'Hare MD, Felmingham D, Gruneberg N. The comparative activity of twelve 4-quinolone antimicrobials against Haemophilus infuenzae and Streptococcus pneumoniae. Drugs Exp Clin Res 1985; 11: 259-62.

14 Chau PY, Leung YK, Ng WWS. Comparative in vitro antibacterial activity of ofloxacin and ciprofloxacin against antibacterial activity of ofioxacin and ciprofloxacin against some selected gram-positive and gram
Infection 1986; 14 (suppl 4): S237-42.

15 Sato K, Inoue Y, Fujii T, Aoyama H, Mitsuhashi S. Antibacterial activity of ofloxacin and its mode of action. Infection 1986; 14: (suppl 4): S226-30.

16 Torres A, Fernandez-Roblas R, Mendez B, Soriano F. Comparative activity of ofloxacin and seven other antimicrobials against urea-splitting microorganisms. Infection 1986; 14 (suppl 4): S233-6.

17 Debbia E, Mannelli S, Gianrossi G, Schito GC. Susceptibility in vitro of gram-positive aerobe and anaerobe bacteria to ofloxacin. Drugs Exp Clin Res 1987; 13: 213-7.

18 Monk JP, Campoli-Richards DM. Ofloxacin: a review of its antibacterial activity, pharmacokinetic properties and its antibacterial activity, pharmacokinetic

19 Periti P, Mazzei T, Nicoletti P. Comparative in vitro activity of ciprofloxacin, ofloxacin and perfloxacin against resistant clinical isolates. Chemioterapia 1987; 6: 75-8.

20 Speciale A, Stefani S, Caccamo R, Nicolosi VM, Nicoletti G. The sensitivity of gram-negative and gram-positive bacteria to ofloxacin. Drugs Exp Clin Res 1987; 12: 555-61.

21 Kiely JS, Schroeder MC, Sesnie JC. New ofloxacin type antibacterial agents. Incorporation of the spiro cyclopropyl group at $\mathrm{N}-1$. F Med Chem 1988; 31: 2004-8.

22 Nagayama A, Nakao T, Taen $H$. In vitro activities of ofloxacin and four other new quinoline-carboxylic acids against Chlamydia trachomatis. Antimicrob Agents Chemother 1988 ; 32: 1735-7.

23 Smith SM. In vitro comparison of A-56619, A-56620 amifloxacin, ciprofloxacin, enoxacin, norfloxacin, and ofloxacin against methicillin-resistant Staphylococcus aureus. Antimicrob Agents Chemother 1986; 29: 325-6.

24 Wise R, Ashby JP, Andrews JM. In vitro activity of PD 127,391 , an enhanced-spectrum quinolone. Antimicrob Agents Chemother 1988; 32:1251-6.

25 Fernandes PB. Mode of action, and in vitro and in vivo activities of the fluoroquinolones. F Clin Pharmacol 1988; 28: 156-68.

26 Crumplin GC, Odell M. Development of resistance to ofloxacin. Drugs 1987; 34 (suppl 1): 1-8.

27 Kresken $M$, Wiedemann B. Development of resistance to nalidixic acid and the fluoroquinolones after the introduction of norfloxacin and ofloxacin. Antimicrob Agents tion of norfloxacin and

28 Mitsui Y, Sakuragi S, Tamura O, Abe M, Watanabe I, Ueno $M$, et al. Effect of ofloxacin ophthalmic solution in the treatment of external bacterial infections of the eye. Foli Ophthalmol 1986; 37: 1115-40.

29 Landis RJ, Heyman ER, Koch GG. Average partia association on three-way contingency tables: a review and discussion of alternative tests. Int Stat Rev 1978; 46: 237-54.

30 Laibson P, Michaud R, Smolin G, Okumoto M, Rosenthal A Cagle G. A clinical comparison of tobramycin and gentamicin sulfate in the treatment of ocular infections. gentamicin sulfate in the treatment

31 Richman J, Zolezio H, Tang-Liu D. Comparison of ofloxacin, gentamicin, and tobramycin concentrations in tears and in
vitro MICs for $90 \%$ of test organisms. Antimicrob Agents vitro MICs for $90 \%$ of test

32 Borrmann L, Tang-Liu D, Kann J, Nista J, Lin ET, Frank J Ofloxacin in human serum, urine, and tear film after topica application. Comea 1992; 11: 226-30.

33 Zhou JS. Ofloxacin content in tears following topical use of $0.3 \%$ ointment. Chung Hua Yen Ko Tsa Chih 1991; 27: 115-7. 